\title{
Evaluation of a serum-based antigen test for tuberculosis in HIV-exposed infants: a diagnostic accuracy study
}

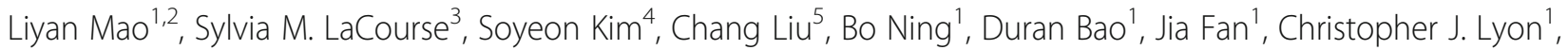
Ziyong Sun ${ }^{2}$, Sharon Nachman ${ }^{6}$, Charles D. Mitchell ${ }^{7^{*}+}$ and Tony Y. Hu ${ }^{1 *+}$

\begin{abstract}
Background: Non-sputum methods are urgently needed to improve tuberculosis diagnosis and treatment monitoring in children. This study evaluated the ability of a serum assay quantifying a species-specific peptide of the Mycobacterium tuberculosis CFP-10 virulence factor via nanotechnology and matrix-assisted laser desorption ionization time-of-flight mass spectrometry to diagnose tuberculosis in HIV-infected and HIV-uninfected infants.

Methods: Serum CFP-10 peptide signal was blinded evaluated in cryopreserved sera of 519 BCG-immunized, HIVexposed infants (284 HIV-infected, 235 HIV-uninfected) from a multi-center randomized placebo-controlled isoniazid prophylaxis trial conducted in southern Africa between 2004 and 2008, who were followed up to 192 weeks for Mtb infection and TB. Children were classified as confirmed, unconfirmed, or unlikely tuberculosis cases using $2015 \mathrm{NIH}$ diagnostic criteria for pediatric TB.

Results: In HIV-infected infants, CFP-10 signal had 100\% sensitivity for confirmed TB (5/5, 95\% Cl, 47.8-100) and 83.7\% sensitivity for unconfirmed TB (36/43, 95\% Cl 69.3-93.2), with 93.1\% specificity (203/218, 95\% Cl 88.9-96.1). In HIV-uninfected infants, CFP-10 signal detected the single confirmed TB case and $75.0 \%$ of unconfirmed TB cases (15/20; 95\% Cl 50.9-91.3), with 96.2\% specificity (177/184, 95\% Cl, 92.3-98.5). Serum CFP-10 achieved 77\% diagnostic sensitivity for confirmed and unconfirmed TB $(13 / 17,95 \% \mathrm{Cl}, 50-93 \%)$ at $\leq 24$ weeks pre-diagnosis, and both CFP-10-positivity and concentration declined following anti-TB therapy initiation.

Conclusions: Serum CFP-10 signal exhibited high diagnostic sensitivity and specificity for tuberculosis in HIVinfected and HIV-uninfected infants and potential utility for early TB detection and monitoring of anti-TB treatment responses.
\end{abstract}

Keywords: Pediatric tuberculosis, CFP-10, Nanotechnology, Mass spectrometry

\footnotetext{
* Correspondence: cmitchel@med.miami.edu; tonyhu@tulane.edu

${ }^{\dagger}$ Charles D. Mitchell and Tony Y. Hu contributed equally to this work.

7Department of Pediatrics, Division of Infectious Diseases and Immunology,

University of Miami Miller School of Medicine, Batchelor Children's Research

Institute, Room 286, 1580 NW 10th Avenue, Miami, FL 33136, USA

${ }^{1}$ Center for Cellular and Molecular Diagnostics, Biochemistry and Molecular

Biology, Tulane University School of Medicine, Room 474, 333 S. Liberty

Street, New Orleans, LA 70112, USA

Full list of author information is available at the end of the article
}

(c) The Author(s). 2021 Open Access This article is licensed under a Creative Commons Attribution 4.0 International License, which permits use, sharing, adaptation, distribution and reproduction in any medium or format, as long as you give appropriate credit to the original author(s) and the source, provide a link to the Creative Commons licence, and indicate if changes were made. The images or other third party material in this article are included in the article's Creative Commons licence, unless indicated otherwise in a credit line to the material. If material is not included in the article's Creative Commons licence and your intended use is not permitted by statutory regulation or exceeds the permitted use, you will need to obtain permission directly from the copyright holder. To view a copy of this licence, visit http://creativecommons.org/licenses/by/4.0/ The Creative Commons Public Domain Dedication waiver (http://creativecommons.org/publicdomain/zero/1.0/) applies to the data made available in this article, unless otherwise stated in a credit line to the data. 


\section{Background}

Approximately one million children develop tuberculosis (TB) and 205,000 die of TB-related causes each year [1]. Eighty percent of these deaths occur in children < 5 years old, with the majority (96\%) of deaths occurring among children who did not receive treatment [2], where missed diagnoses are likely responsible for undertreatment. Children with $\mathrm{TB}$, particularly infants, frequently have paucibacillary TB, exhibit non-specific symptoms, and are likely to rapidly progress to disseminated or extrapulmonary $\mathrm{TB}$ in the absence of appropriate treatment [3-6]. This clinical presentation, combined with difficulty obtaining respiratory samples, makes it challenging to diagnose pediatric $\mathrm{TB}$ and monitor treatment responses using standard sputum-based methods $[3,7,8]$.

The gold-standard of mycobacterial culture is positive in only $30-62 \%$ of pediatric TB cases $[8,9]$. Molecular diagnostic assays such as Xpert MTB/RIF and Xpert MTB/RIF Ultra enable rapid diagnosis but are less sensitive in children [10-14]. Immunological tests (e.g., tuberculin skin tests (TSTs) and interferon gamma-release assays) detect the immune response to Mycobacterium tuberculosis (Mtb), but do not distinguish $M t b$ infection from $\mathrm{TB}$, and are less sensitive in children with compromised or immature immune systems $[15,16]$. However, direct detection of circulating $M t b$-derived factors, such as the virulence factors CFP-10 and ESAT-6 [17], can be hampered by low levels and masking effects [18], and current direct detection assays have demonstrated poor sensitivity in identifying active TB [19-21].

We recently developed a novel assay applying nanotechnology and matrix-assisted laser desorption ionization time-of-flight (MALDI-TOF) mass spectrometry to directly detect an $M t b$-specific CFP-10 peptide (CFP-10pep) in trypsin-digested sera, allowing $\mathrm{TB}$ diagnosis from a small blood volume $(100 \mu \mathrm{L})$. This assay diagnosed pulmonary TB with high sensitivity in culture-confirmed HIV-negative (93\%) and HIV-positive (91\%) adults (specificity 87$100 \%$ ) and detected serum CFP-10pep in extrapulmonary and culture-negative TB cases $[18,22,23]$. This assay uses fresh or frozen serum, allowing samples to be transported to and analyzed at central testing sites without restricting the time available to obtain valid assay results. Non-sputum diagnostic methods that utilize small blood volumes could be of particular benefit to infants, in whom sputum confirmation is typically lacking at diagnosis and during treatment. Herein, we evaluated the diagnostic performance of this assay for pediatric $\mathrm{TB}$, using stored sera from HIV-exposed infants enrolled in a TB prevention trial.

\section{Methods}

\section{Participants and study design}

De-identified serum and clinical data were obtained from IMPAACT P1041, a multi-center randomized, doubleblind, placebo-controlled trial conducted in southern Africa between 2004 and 2008 that compared pre-exposure isoniazid (INH) prevention therapy to placebo in infants with perinatal HIV exposure [24]. Infants meeting P1041 enrollment criteria (see Additional file) were randomized to daily INH or placebo for 96 weeks after obtaining informed consent from their legal guardians, segregated into HIV-infected and HIV-uninfected subgroups, and evaluated for up to 96 weeks after intervention. HIV-infected infants were scheduled for study visits every 12 weeks, but HIV-uninfected infant visits were reduced to every 24 weeks after their intervention period. Infants were evaluated for TB by the P1041criteria at all visits using all diagnostic evidence (see Additional file), and sera remaining from blood assays was archived.

P1041 participants were eligible for the current study if they had complete demographic information, results from all conducted clinical tests, and at least one analyzable serum sample after excluding hemolyzed, hyperlipidemic, and low volume $(<100 \mu \mathrm{L})$ samples. Serum was analyzed by personnel who were blinded to all clinical and TB classification data using a previously reported assay employing nanoparticle-based immuneenrichment and MALDI-TOF mass spectrometry to detect and quantify CFP-10pep signal, as described below. Statistical analyses were performed using data from a locked database containing clinical information and CFP-10pep results. All study personnel committed to the research plan and to ensuring the accuracy and completeness of the data analysis.

\section{Case classification}

Infants were categorized post-hoc by investigators with pediatric TB expertise (CDM, SML) blinded to CFP10pep results using $2015 \mathrm{NIH}$ consensus criteria for pediatric TB diagnosis (hereafter $2015 \mathrm{NIH}$ criteria) [25]. Infants were categorized as confirmed TB ( $M t b$ culturepositive), unconfirmed TB ( $\geq 2$ non-bacteriologic forms of TB evidence), or unlikely TB (no/insufficient evidence of TB or a confirmed alternative diagnosis) [25]. Unconfirmed and unlikely TB cases were categorized by the presence or absence of immunologic evidence of $M t b$ infection (TST result; see Additional file). Extrapulmonary TB cases were identified using the P1041 criteria [ 24].

\section{Serum CFP-10pep assays}

Serum CFP-10pep was analyzed using a nanoparticlebased immunoenrichment assay read by MALDI-TOF mass spectrometry (see Additional file) that detects $M t b$-specific CFP-10pep from trypsin-digested serum or 
EDTA plasma samples [18, 22, 23]. Samples producing CFP-10pep peaks ( $\mathrm{m} / \mathrm{z} 1593.75)$ with signal-to-noise ratios $\geq 3$ were considered CFP-10pep-positive, and CFP10pep signal was analyzed as a ratio against a spiked sequence-matched isotope-labeled internal standard (IS) peptide $(\mathrm{m} / \mathrm{z}$ 1603.60).

CFP-10pep diagnostic sensitivity estimates utilized the serum sample collected nearest TB diagnosis and within \pm 24 weeks of diagnosis by $2015 \mathrm{NIH}$ criteria $( \pm 1$ HIVuninfected cohort post-intervention study visit), since few infants had serum available at their post-hoc diagnosis visit. CFP-10pep diagnostic specificity estimates analyzed all available serum from unlikely $\mathrm{TB}$ cases, and participants were considered CFP-10pep-negative only if all their samples were CFP-10pep-negative.

CFP-10pep changes pre-TB diagnosis and posttreatment initiation were analyzed using all samples from confirmed and unconfirmed TB cases, grouping CFP-10pep results by 12-week intervals, and comparing CFP-10pep changes among TB cases with and without positive treatment responses (resolution/improvement of TB signs/symptoms within 3 months of treatment initiation without new signs/symptoms).

\section{Statistical analyses}

Statistical analyses employed IBM SPSS version 24.0, and GraphPad Prism 7 for chi-square and Fisher exact tests, one-way ANOVAs with Bonferroni's posttests, and Kruskal-Wallis one-way ANOVAs with Dunn's post-tests, as determined by sample distribution and variance. Sensitivity and specificity are reported with Clopper-Pearson 95\% CIs. Differences were considered statistically significant for two-sided tests with $p$ values $<0.05$.

\section{Results}

\section{Study population and baseline characteristics}

Of the 1351 enrolled P1041 infants, 626 completed follow-up and had archived serum samples, and 519 remained after excluding all those with missing clinical information or with unanalyzable serum samples (Fig. 1). Differences between included and excluded infants were primarily minor (Additional file: Table S1), although HIV-infected children were excluded at higher rates and included children exhibited higher rates of TB regardless of their HIV infection status. Median age at enrollment was 94 days, $45.3 \%$ were HIV-infected, and $46.6 \%$ were male (Table 1). Half the infants diagnosed with TB by 2015 NIH criteria developed TB by 15 months of age (median 48 weeks post-enrollment; Additional file: Fig. S1A), which differed between HIV-infected and HIVuninfected infants (median 36 versus 72 weeks, $p=$ 0.008; Additional file: Fig. S1B-C).
The proportion of TB cases (confirmed and unconfirmed) was similar between HIV-infected and HIVuninfected infants $(23.2 \%$ versus $21.7 \%, p=0.75)$ as were diagnosis distributions (Table 2). Most TB cases were pulmonary $\mathrm{TB}$, but five HIV-infected infants had extrapulmonary $\mathrm{TB}$, with two having both pulmonary and extrapulmonary TB. The distribution of infants who received INH or placebo did not differ by HIV status and TB category (Additional file: Table S2). Most TB cases (86.3\%, 101/117) had Mtb culture, smear, chest X-ray, and TST data, but were primarily diagnosed by chest Xray and TST (Additional file: Fig. S2).

\section{CFP-10pep diagnostic performance for pediatric TB}

Samples suitable for CFP-10pep analysis were limited in number and distribution (Additional file: Table S3 and Table S4), and few infants had serum available at their retrospective diagnosis by $2015 \mathrm{NIH}$ criteria (14\% and $8 \%$ of HIV-infected and HIV-uninfected infants). We therefore analyzed the serum sample that was dawn nearest TB diagnosis $( \pm 1$ post-intervention study visit for the HIV-uninfected cohort) to calculate CFP-10pep diagnostic sensitivity, since most confirmed $(75 \% ; 6 / 8)$ and unconfirmed $(57.8 \% ; 63 / 109)$ TB cases had serum within this interval. Children with and without serum in this window revealed similar clinical characteristics (Additional file: Table S5 and Fig. S2), suggesting the absence of selection bias.

Serum CFP-10pep demonstrated 95\% overall specificity (22/402, Table 3), which ranged from 85.7 to $100 \%$ in the unlikely TB subgroups (Table 4 ), and robust sensitivity for confirmed $(100 \% ; 6 / 6)$ and unconfirmed $(81 \%$; 51/63) TB cases diagnosed by clinical evidence (Table 3; Additional file: Table S6). HIV-infected and HIVuninfected infants with unconfirmed TB had similar CFP-10pep diagnostic sensitivities (84\% versus 75\%; $p=$ 0.50 ) and specificities (93\% versus $96 \%, p=0.19$ ), which differed in HIV-uninfected infants only with evidence of $M t b$ infection ( $86 \%$ versus $98 \%, p=0.03$, Table 3 ). CFP10pep was not detected in most unlikely TB cases with evidence of $M t b$ infection (88.7\%), including HIVinfected (90.6\%) and HIV-uninfected (85.7\%) individuals (Table 3). INH prophylaxis did not appear to affect CFP10pep sensitivity for confirmed and unconfirmed TB or specificity for unlikely $\mathrm{TB}$, when analyzed overall or by HIV- or $M t b$-infection status (Additional file: Tables S7; Table S8).

CFP-10pep signal was higher in TB versus unlikely TB cases in both HIV-infected $(1.9 \pm 0.3$ versus $0.1 \pm 0.03$, $p<0.001)$ and HIV-uninfected infants $(1.4 \pm 0.4$ versus $0.09 \pm 0.04, p<0.001$; Fig. 2a), but did not differ with evidence of $M t b$ infection or with pulmonary and extrapulmonary TB (Additional file: Fig. S3A-B). 


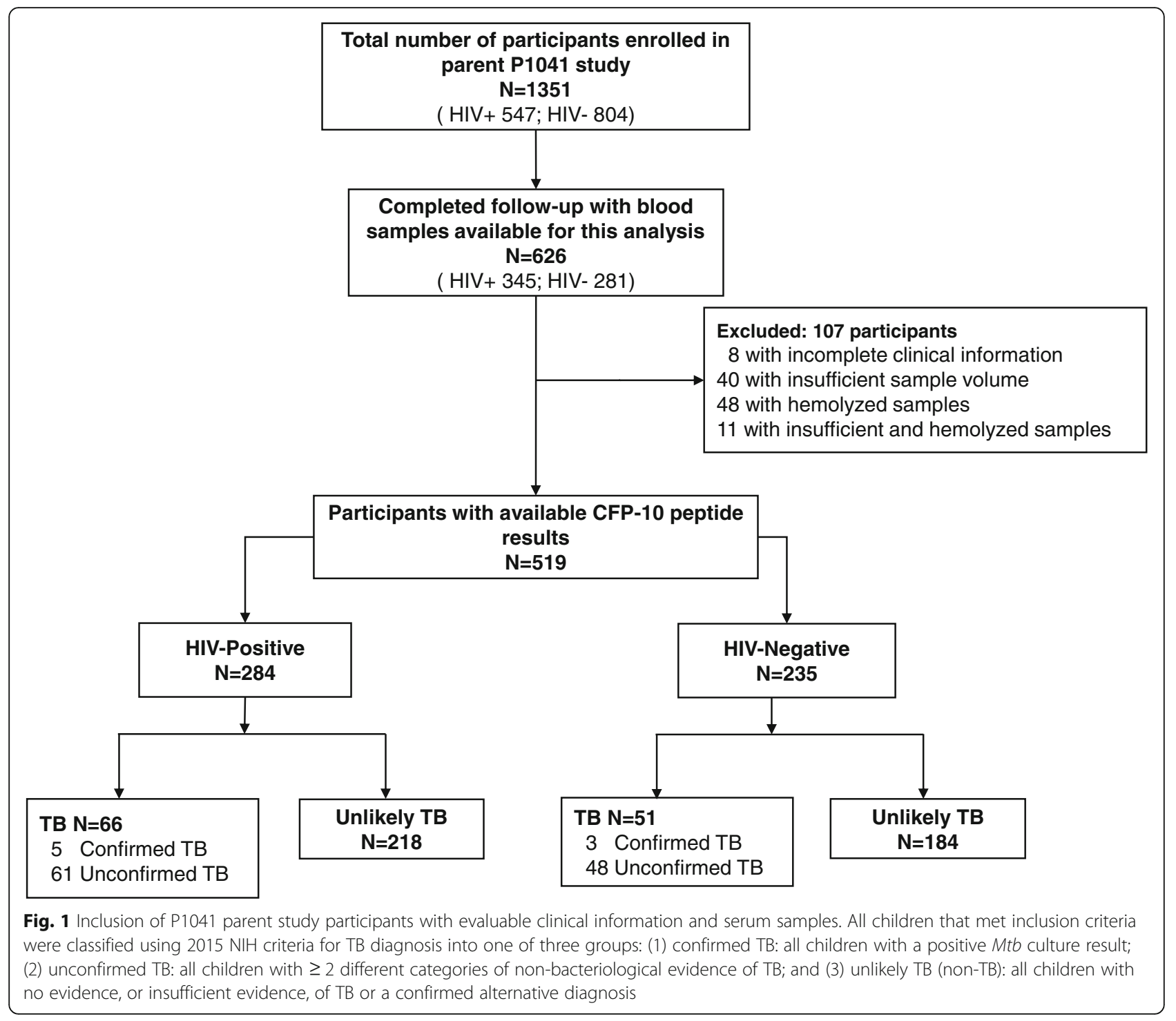

CFP-10pep signal more accurately distinguished TB and unlikely TB than $M t b$ culture $(83.8 \%$ versus $61.0 \%$, $p<0.001)$, AFB smear $(82.8 \%$ versus $55.7 \%, p<0.001$ ), TST $(91.7 \%$ versus $80.3 \%, p=0.007)$, and chest $\mathrm{X}$-ray $(84.1 \%$ versus $79.7 \%, p=0.015)$ results in infants with each result (Table 5). Serum CFP-10pep detected 45 TB cases not identified by culture or smear (Fig. 2b) and had higher sensitivity for pulmonary and extrapulmonary TB than culture or smear (Fig. 2c, d).

CFP-10pep for early TB detection and treatment response Most TB cases with pre-diagnosis serum had at least one CFP-10pep-positive sample prior to diagnosis (60\%; $32 / 53$ ), with positive samples clustering near diagnosis, but detectable by 60 weeks before diagnosis (median 18 weeks pre-diagnosis) (Fig. 3a). CFP-10pep detection rates tended to be higher in HIV-infected TB cases, but similar detection time courses were observed regardless of HIV infection status (Additional file: Fig. S3C-D). Overall, CFP-10pep positive sample frequency rose to $46 \%$ by 36 weeks pre-diagnosis, ranged between $76-83 \%$ and $75-100 \%$ in the 24 -week intervals pre- and postdiagnosis, respectively and then decreased to $18-43 \%$ (Additional file: Fig. S4A). Culture and smear positive rates were low (12\% and $13 \%)$ at diagnosis and zero by 48 weeks post-treatment initiation. Similar results were observed for HIV-infected and HIV-uninfected infants (Additional file: Fig. S4B-C). Six infants had recurrent TB with 5- to 96-week intervals between first TB resolution and second TB diagnosis (Fig. 3b). Five were CFP10pep-positive at or after disease recurrence (Fig. 3b; $\# 1-5$ ), including one patient who completed treatment without recorded evidence for a cure or continued TB disease (Fig. 3b, \#5).

Most TB cases identified and treated under the P1041 protocol were also identified by $2015 \mathrm{NIH}$ criteria (91\%; 
Table 1 Baseline demographics and clinical characteristics of study participants by HIV and TB status

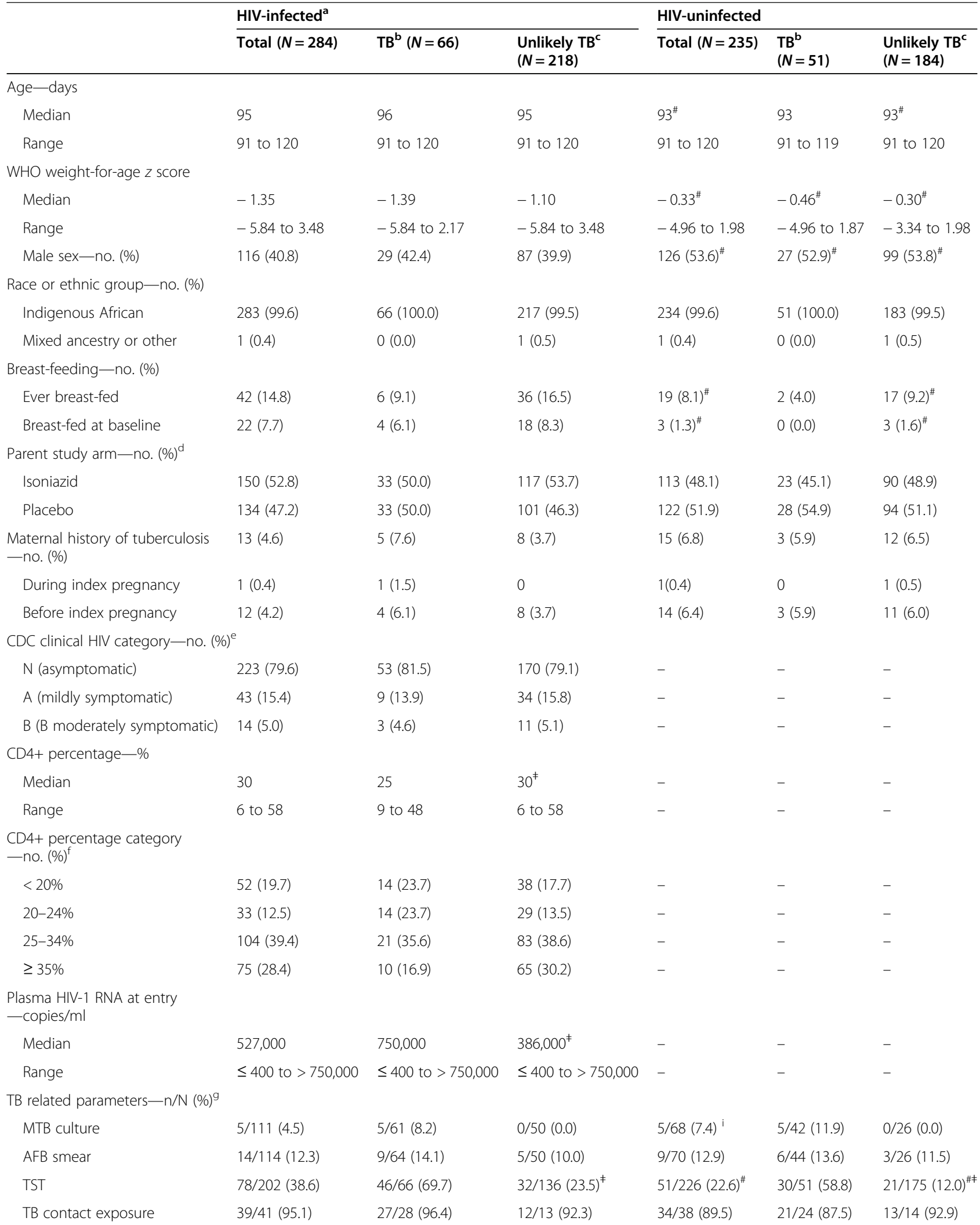


Table 1 Baseline demographics and clinical characteristics of study participants by HIV and TB status (Continued)

\begin{tabular}{|c|c|c|c|c|c|c|}
\hline & \multicolumn{3}{|l|}{ HIV-infected $^{\mathrm{a}}$} & \multicolumn{3}{|l|}{ HIV-uninfected } \\
\hline & Total $(N=284)$ & $\mathrm{TB}^{\mathrm{b}}(N=66)$ & $\begin{array}{l}\text { Unlikely TB' } \\
(N=218)\end{array}$ & Total $(N=235)$ & $\begin{array}{l}\mathrm{TB}^{\mathrm{b}} \\
(N=51)\end{array}$ & $\begin{array}{l}\text { Unlikely TB' } \\
(N=184)\end{array}$ \\
\hline Chest X-ray & $75 / 134(56.0)$ & $58 / 66$ (87.9) & $17 / 68(25.0)^{\ddagger}$ & $61 / 98(62.2)$ & $49 / 51(96.1)$ & $12 / 47(25.5)^{\ddagger}$ \\
\hline $\begin{array}{l}\text { Signs/symptoms consistent } \\
\text { with TB }\end{array}$ & 13/284 (4.6) & 10/66 (15.2) & $3 / 218(1.4)^{\ddagger}$ & $11 / 235(4.7)$ & 6/51 (11.8) & $5 / 184(2.7)^{\ddagger}$ \\
\hline $\begin{array}{l}\text { Positive response to anti-TB } \\
\text { therapy }^{\mathrm{h}}\end{array}$ & $38 / 77$ (49.4) & $37 / 60(61.7)$ & $1 / 17(5.9)^{\ddagger}$ & $33 / 61(54.1)$ & $32 / 46(69.6)$ & $1 / 15(6.7)^{\ddagger}$ \\
\hline
\end{tabular}

HIV status was determined following enrollment; TB status was retroactively applied to the study population using the $2015 \mathrm{NIH}$ criteria

Symbols denote $p$ values $<0.05$ for differences between the TB and unlikely TB groups ( $)$ ) and the HIV-infected and HIV-uninfected groups (\#)

${ }^{a}$ Two participants were HIV-uninfected at entry but tested HIV-infected approximately 24 weeks after enrollment and therefore classified as HIV-infected

${ }^{b}$ Participants with confirmed TB (Mtb culture-positive) and unconfirmed TB ( $\geq 2$ types of non-bacteriological evidence of TB) evaluated as a group since the size of the confirmed TB group ( $N=8$; see Fig. 1) prevent meaningful comparisons with other groups

'Unlikely TB: children never suspected of TB or suspected of TB but with no, or insufficient, evidence for TB diagnosis, or with a confirmed alternative diagnosis

${ }^{d}$ Children enrolled in the P1041 parent study received INH prophylaxis for up to 96 weeks or until achieving a primary endpoint (first occurrence of TB disease or

$M+b$ infection, or death from any cause) to evaluate whether primary INH prophylaxis improved TB disease-free survival among HIV-infected children or Mtb

infection-free survival among HIV-uninfected children in a population immunized with the BCG vaccine

${ }^{\mathrm{e}}$ Four participants with missing data and HIV-uninfected participants at entry were not included from the percentages

$\mathrm{f}_{20}$ participants with missing data and HIV-uninfected participants at entry were not included from the percentages

${ }^{9} \mathrm{n} / \mathrm{N}(\%)$ indicate the number of children with positive results per the total number of children with this information and the percent of children with a

positive result

${ }^{h}$ Children classified as TB cases (definite, probable, and possible TB) according to the P1041 protocol or as having "non-algorithm TB" by clinical experts were started on anti-TB treatment. The majority of children identified as TB cases in the P1041 study were also categorized as TB cases by the 2015 NIH criteria (Table S10)

'Two TB patients with culture positive results were first diagnosed as unconfirmed TB

106/117) and had positive treatment responses $(66 \% ; 70 /$ 106) that did not differ by HIV status (Tables $1,62 \%$ vs. $70 \%, p>0.05)$. Among those with pre- and postdiagnosis serum, $60 \%(15 / 25)$ responded to treatment and tended to exhibit CFP-10pep decreases not observed in non-responders (Fig. 3c). CFP-10pep signal also increased near TB diagnosis, and decreased to nondetectable levels during or after anti-TB treatment, consistent with clinical evaluation of disease resolution (Additional file: Fig. S5).

\section{Discussion}

This study extends our previous research employing serum CFP-10pep assays to diagnose TB in adults [18, $22,23]$, indicating that it can effectively diagnose TB in HIV-infected and HIV-uninfected infants, including unconfirmed TB cases missed by sputum-based methods. Our data also suggests its potential utility for diagnosis of nascent TB cases and monitoring treatment responses.

The World Health Organization (WHO) recommends that non-sputum biomarker tests have sensitivities $\geq 66 \%$

Table 2 Summary of groups present in current study

\begin{tabular}{|c|c|c|c|c|}
\hline & Total $*(N=519)$ & HIV-infected $(N=284)$ & HIV-uninfected $(N=235)$ & $P$ value \\
\hline TB cases-no. (\%) & $117(22.5)$ & $66(23.2)^{*}$ & $51(21.7)$ & 0.68 \\
\hline Confirmed PTB & $6(1.2)$ & $3(1.1)$ & $3(1.3)$ & 0.26 \\
\hline Confirmed EPTB ${ }^{a}$ & $2(0.4)$ & $2(0.7)$ & - & - \\
\hline Unconfirmed PTB & $108(20.8)$ & $60(21.1)$ & $48(20.4)$ & - \\
\hline MTB infection ${ }^{\mathbf{b}}$ & $73(14.1)$ & $45(15.8)$ & $28(11.9)$ & 0.10 \\
\hline No MTB infection ${ }^{c}$ & $35(6.7)$ & $15(5.3)$ & $20(8.5)$ & - \\
\hline Unconfirmed EPTB ${ }^{d}$ & $3(0.6)$ & $3(1.1)$ & - & - \\
\hline Unlikely TB—no. (\%) & $402(77.5)$ & $218(76.8)$ & $184(78.3)$ & - \\
\hline MTB infection & $53(10.2)$ & $32(11.3)$ & $21(8.9)$ & 0.33 \\
\hline No MTB infection ${ }^{\mathbf{e}}$ & $349(67.3)$ & $186(65.5)$ & $163(69.4)$ & - \\
\hline Died & $9(1.7)$ & $7(2.5)$ & $2(0.9)$ & 0.16 \\
\hline
\end{tabular}

*Two infants diagnosed with both unconfirmed EPTB and unconfirmed PTB were counted once in this total

${ }^{a}$ Both of these EPTB cases were diagnosed as TB lymphadenitis

${ }^{\mathrm{b}}$ MTB infection = TST-positive (induration of $\geq 5 \mathrm{~mm}$ for HIV-infected infants and $\geq 10 \mathrm{~mm}$ for HIV-uninfected infants)

${ }^{c}$ No MTB infection = TST-negative (induration of $<5 \mathrm{~mm}$ for HIV-infected infants and $<10 \mathrm{~mm}$ for HIV-uninfected infants)

${ }^{\mathrm{d}}$ Two infants were diagnosed with both unconfirmed TB lymphadenitis and unconfirmed PTB, and the third was diagnosed with TB meningitis

'TST-negative or no TST result available for infants not suspected of TB who did not reach a protocol-designated visit for TST performance (parent study weeks $96,144,196)$ 
Table 3 Diagnostic performance of CFP-10pep assay for TB

\begin{tabular}{|c|c|c|c|c|c|c|c|}
\hline & \multirow[t]{2}{*}{ Confirmed $\mathrm{TB}^{\mathrm{a}}$} & \multicolumn{3}{|c|}{ Unconfirmed $\mathrm{TB}^{\beta}$} & \multicolumn{3}{|l|}{ Unlikely $\mathrm{TB}^{Y}$} \\
\hline & & $\begin{array}{l}\text { MTB } \\
\text { infection }^{a}\end{array}$ & $\begin{array}{l}\text { No MTB } \\
\text { infection }^{\text {b }}\end{array}$ & Total & $\begin{array}{l}\text { MTB } \\
\text { infection }\end{array}$ & $\begin{array}{l}\text { No MTB } \\
\text { infection }^{c}\end{array}$ & Total \\
\hline \multicolumn{8}{|l|}{ All participants $(N=471)$} \\
\hline CFP-10pep +, no. (\%) & $6(100.0)$ & $34(79.1)$ & $17(85.0)$ & $51(81.0)$ & $6(11.3)$ & $16(4.6)$ & $22(5.5)$ \\
\hline CFP-10pep -, no. (\%) & - & $9(20.9)$ & $3(15.0)$ & $12(19.0)$ & $47(88.7)$ & $333(95.4)$ & $380(94.5)$ \\
\hline Sensitivity—\% (95\% Cl) & $100(54.1-100.0)$ & $79.1(64.0-90.0)$ & $85.0(62.1-96.8)$ & $81.0(69.1-89.8)$ & - & - & - \\
\hline Specificity_\% (95\% Cl) & - & - & - & - & $88.7(77.0-95.7)$ & $95.4(92.7-97.4)$ & $94.5(91.8-96.5)$ \\
\hline \multicolumn{8}{|c|}{ HIV-infected participants $(N=266)$} \\
\hline CFP-10pep +, no. (\%) & $5(100.0)$ & $24(80.0)$ & $12(92.3)$ & $36(83.7)$ & $3(9.4)$ & $12(6.4)$ & $15(7.7)$ \\
\hline CFP-10pep -, no. (\%) & - & $6(20.0)$ & $1(7.7)$ & $7(16.3)$ & $29(90.6)$ & $174(93.6)$ & $203(92.3)$ \\
\hline Sensitivity—\% (95\% Cl) & $100(47.8-100.0)$ & $80.0(61.4-92.3)$ & $92.3(64.0-99.8)$ & $83.7(69.3-93.2)$ & - & - & - \\
\hline Specificity—\% (95\% Cl) & - & - & - & - & $90.6(75.0-98.0)$ & $93.6(89.0-96.6)$ & $93.1(88.9-96.1)$ \\
\hline \multicolumn{8}{|c|}{ HIV-uninfected participants $(N=205)$} \\
\hline CFP-10pep +, no. (\%) & $1(100.0)$ & $10(76.9)$ & $5(71.4)$ & $15(75.0)$ & $3(14.3)$ & $4(2.4)$ & $7(4.3)$ \\
\hline CFP-10pep -, no. (\%) & - & $3(23.1)$ & $2(28.6)$ & $5(25.0)$ & $18(85.7)$ & $159(97.6)$ & $177(96.2)$ \\
\hline Sensitivity—\% (95\% Cl) & $100(2.5-100.0)$ & $76.9(46.2-95.0)$ & $71.4(29.0-96.3)$ & $75.0(50.9-91.3)$ & - & - & - \\
\hline Specificity—\% (95\% Cl) & - & - & - & - & $85.7(63.7-97.0)$ & $97.6(93.8-99.3)$ & $96.2(92.3-98.5)$ \\
\hline \multicolumn{8}{|c|}{ 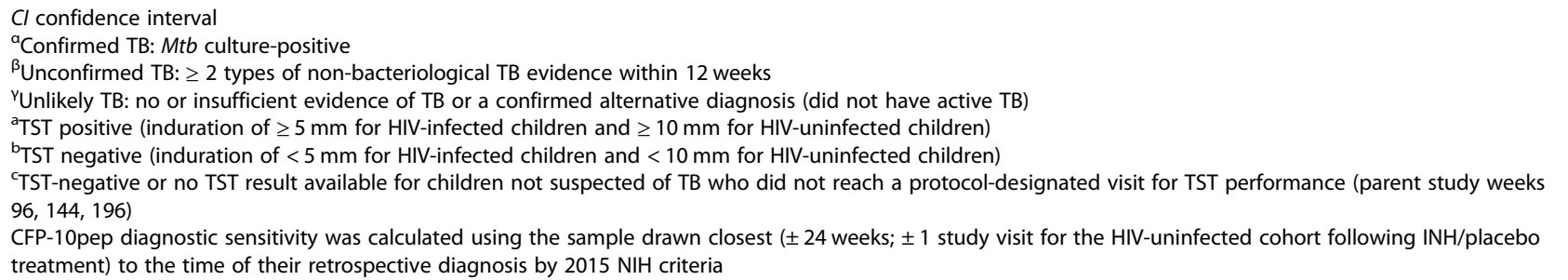 } \\
\hline
\end{tabular}

for children with intrathoracic confirmed TB cases to match Xpert MTB/RIF sensitivity [26]. CFP-10pep demonstrated 100\% (95\% CI, 54-100\%) and 81\% (95\% CI, 69-90\%) sensitivity for confirmed and unconfirmed TB and unlike most other TB diagnostics did not differ by HIV status, in agreement with previous results with adult TB cases $[18,22,26]$. Serum CFP-10pep signal was detected in most unconfirmed TB cases (> 80\%; 51/63), and all (5/5) extrapulmonary TB cases, including TB lymphadenitis and TB meningitis cases missed by respiratory sampling.

Serum CFP-10pep exhibited 95\% overall specificity (95\% CI 92-97\%), approaching the 98\% specificity threshold recommended by the WHO, but varied among

Table 4 CFP-10pep specificity in subgroups of the unlikely TB population

\begin{tabular}{|c|c|c|c|c|c|c|}
\hline \multirow[t]{2}{*}{ Unlikely TB } & \multicolumn{2}{|l|}{ Total } & \multicolumn{2}{|l|}{ HIV-infected } & \multicolumn{2}{|c|}{ HIV-uninfected } \\
\hline & $\begin{array}{l}\text { CFP-10 } \\
\text { (pos./total) }\end{array}$ & $\begin{array}{l}\text { Specificity \% } \\
(95 \% \mathrm{Cl})\end{array}$ & $\begin{array}{l}\text { CFP-10 } \\
\text { (pos./total) }\end{array}$ & $\begin{array}{l}\text { Specificity \% } \\
(95 \% \mathrm{Cl})\end{array}$ & $\begin{array}{l}\text { CFP-10 } \\
\text { (pos./total) }\end{array}$ & $\begin{array}{l}\text { Specificity \% } \\
(95 \% \mathrm{Cl})\end{array}$ \\
\hline NTM infection & $2 / 14$ & $85.7(57.2-98.2)$ & $1 / 12$ & $91.7(61.5-99.8)$ & $1 / 2$ & $50.0(1.3-98.7)$ \\
\hline Other infections* & $13 / 153$ & $91.5(85.9-95.4)$ & $11 / 88$ & $87.5(78.7-93.6)$ & $2 / 65$ & $96.9(89.3-99.6)$ \\
\hline Other inflammatory diseases & $7 / 148$ & $95.3(90.5-98.1)$ & $3 / 63$ & $95.2(86.7-99.0)$ & $4 / 85$ & $95.3(88.4-98.7)$ \\
\hline Other diseases & $0 / 35$ & $100(90.0-100)$ & $0 / 28$ & $100(87.7-100)$ & $0 / 7$ & $100(59.0-100)$ \\
\hline Latent TB infection & $5 / 44$ & $88.6(75.4-96.2)$ & $3 / 25$ & $88.0(68.8-97.5)$ & $2 / 19$ & $89.5(66.9-98.7)$ \\
\hline No disease (healthy) & $1 / 45$ & $97.8(88.2-99.9)$ & $1 / 27$ & $96.3(81.0-99.9)$ & $0 / 18$ & $100(81.5-100)$ \\
\hline Unclear diagnosis & $0 / 5$ & $100(47.8-100)$ & $0 / 1$ & $100(2.5-100)$ & $0 / 4$ & $100(39.8-100)$ \\
\hline Total & $22 / 402^{\#}$ & $94.5(91.8-96.5)$ & $15 / 218$ & $93.1(88.9-96.1)$ & $7 / 184$ & $96.2(92.3-98.5)$ \\
\hline
\end{tabular}

*Bacterial, viral, fungal, and other infections

\#Several children had co-infections, including 7 with non-tuberculous mycobacterium (NTM) infections and 12 with latent TB infections, while 23 children with latent TB infections also had an unrelated inflammatory disease 


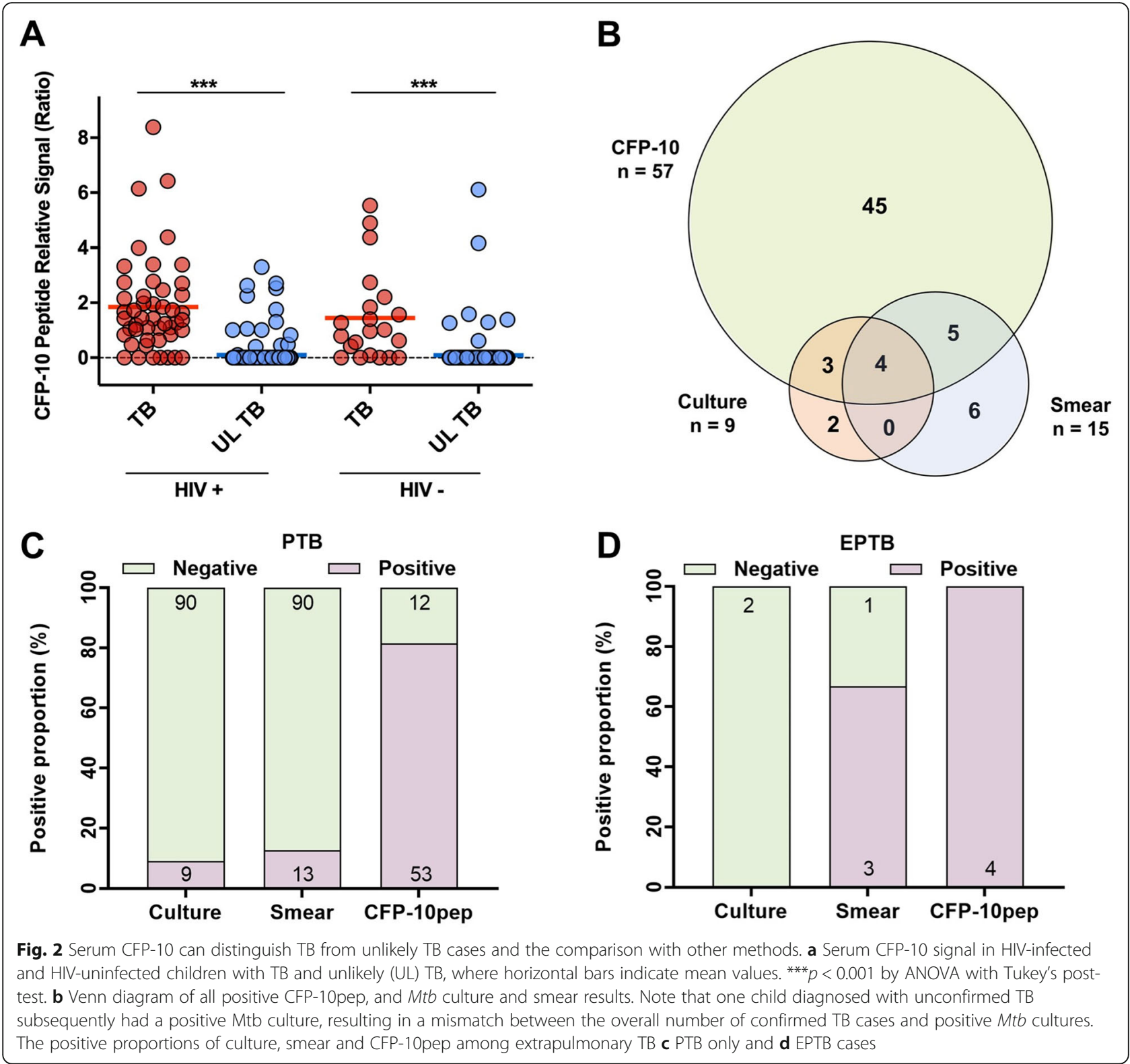

Table 5 TB diagnostic performance of CFP-10pep, smear, culture, and TST

\begin{tabular}{|c|c|c|c|c|c|c|c|c|c|c|}
\hline Comparison & Participants $^{\mathrm{a}}$ & $\begin{array}{l}\text { CFP-10 } \\
\text { Sensitivity }\end{array}$ & $\begin{array}{l}2^{\text {nd }} \text { assay } \\
\text { Sensitivity }\end{array}$ & $p$ value $^{b}$ & $\begin{array}{l}\text { CFP-10 } \\
\text { Specificity }\end{array}$ & $\begin{array}{l}2^{\text {nd }} \text { assay } \\
\text { Specificity }\end{array}$ & $p$ value & $\begin{array}{l}\text { CFP-10 } \\
\text { Accuracy }\end{array}$ & $\begin{array}{l}2^{\text {nd }} \text { assay } \\
\text { Accuracy }\end{array}$ & $p$ value \\
\hline $\begin{array}{l}\text { CFP-10pep } \\
\text { vs X-ray }\end{array}$ & 183 & $82.3 \%$ (81.9-82.7) & $88.2 \%(87.9-88.5)$ & 0.346 & $85.2 \%(85.0-85.4)$ & $74.7 \%(74.4-75.1)$ & 0.019 & $84.1 \%(84.0-84.2)$ & $79.7 \%(79.6-79.9)$ & 0.015 \\
\hline $\begin{array}{l}\text { CFP-10pep } \\
\text { vs smear }\end{array}$ & 140 & $85.9 \%$ (85.5-86.3) & $15.6 \%(15.2-16.0)$ & $<0.001$ & $80.2 \%(79.8-80.6)$ & $89.4 \%(89.2-89.7)$ & 0.127 & $82.8 \%(82.6-83.0)$ & $55.7 \%(55.3-56.0)$ & $<0.001$ \\
\hline $\begin{array}{l}\text { CFP-10pep } \\
\text { vs culture }\end{array}$ & 136 & $88.3 \%$ (87.9-88.6) & $11.6 \%(11.3-12.0)$ & $<0.001$ & $80.2 \%(79.8-80.6)$ & $100 \%(95.3-100)$ & $<0.001$ & $83.8 \%(83.6-84.0)$ & $61.0 \%(60.6-61.37)$ & $<0.001$ \\
\hline $\begin{array}{l}\text { CFP-10pep } \\
\text { vs TST }\end{array}$ & 377 & $81.8 \%(81.3-82.2)$ & $68.1 \%(67.5-68.8)$ & 0.083 & $93.8 \%(93.8-93.9)$ & $82.9 \%(82.8-83.0)$ & $<0.001$ & $91.7 \%(91.7-91.8)$ & $80.3 \%(80.2-80.4)$ & 0.007 \\
\hline
\end{tabular}

The results listed in this Table indicate percent diagnostic sensitivity, specificity and accuracy with an estimated $95 \% \mathrm{Cl}$ adjusted for sample size

a Participants who were conducted both CFP-10pep assay and secondary assay (X-ray, smear, culture, and TST, respectively)

${ }^{b}$ Listed $p$ values indicate the probability for a significant difference between the diagnostic sensitivity, specificity, or accuracy of CFP-10 and the test listed in the first column of the matching row by McNemar's test 

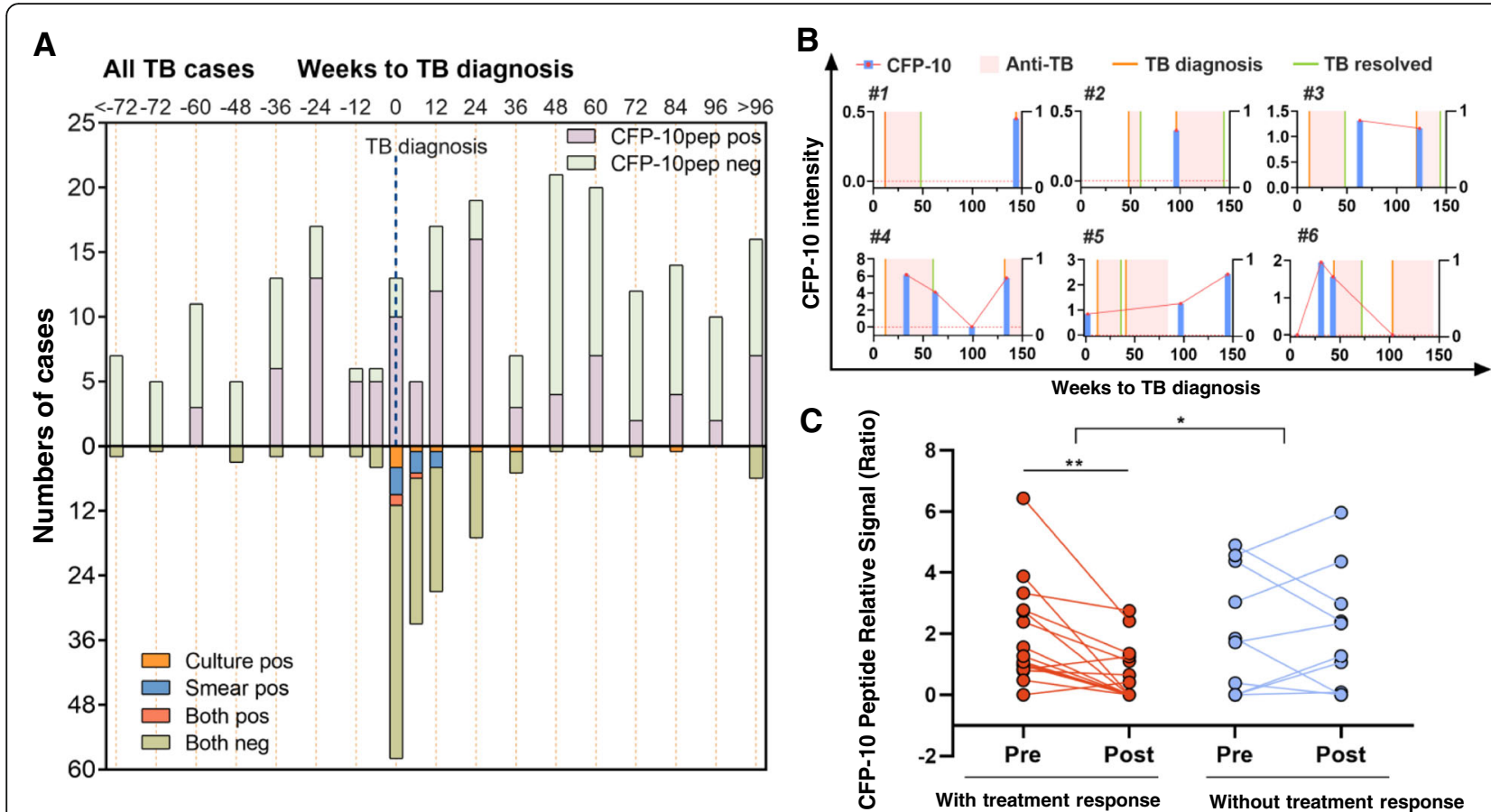

Fig. 3 Serum CFP-10pep assay in early detection, recurrent TB, and TB treatment monitoring. a Distribution of CFP-10pep-positive and CFP10 pep-negative signal (above the $x$-axis) and culture and/or smear positive and culture + smear negative results (below the $x$-axis) in total TB cases, where time zero (vertical dashed line) denotes time of TB diagnosis. Only one sample per child was available and evaluated in each 12week interval. b Recurrent TB cases. The orange vertical lines denote time of TB diagnosis relative to study enrollment, the pink box indicates the duration of an anti-TB treatment, and the vertical green lines indicate the time of TB resolution, while the red dots and blue bar connected by red lines indicate the time and intensity of serum CFP-10pep signal. c Serum CFP-10pep signal changes from pre-diagnosis (pre) to postdiagnosis (post) in TB cases with (red dots) and without (blue dots) positive anti-TB treatment responses. Connected points indicate the magnitude and time frame of changes for individual patients. ${ }^{*} p<0.01$ by paired $t$ test, ${ }^{*} p<0.05$ by repeated measure mixed model to compare the paired difference between TB cases with and without positive anti-TB treatment responses

unlikely TB subgroups, ranging from 85.7 to $88.6 \%$ in non-tuberculous mycobacteria (NTM) and latent TB infection subgroups and from 97.8 to $100 \%$ in subgroups without and with other disease (Table 4). The two false positives NTM CFP-10pep results matched a Mycobacterium kansasii infection, which could express a CFP-10 ortholog, and a Mycobacterium avium and bacterial/fungal co-infection. Most CFP-10pep false positives in the latent TB subgroup ( 4 of 5) were detected 6 to 60 weeks before a positive TST and could indicate nascent $M t b$ infections that subsequently resolved to latent TB infections. Notably, most unlikely TB cases with CFP-10pep positive serum (73\%) met at least one criterion for unconfirmed TB diagnosis, suggesting that some could have had subclinical disease. CFP-10pep signal might thus identify individuals who might benefit from anti-TB intervention, particularly in populations where latent $\mathrm{TB}$ cases do not routinely receive anti-TB treatment.

Delayed anti-TB treatment initiation, especially for children with HIV/TB co-infections or disseminated TB (including TB meningitis), may result in irreversible damage or death [2]. Serum CFP-10pep was detected up to 60 weeks before TB diagnosis, and identified TB with
$77 \%$ sensitivity 24 weeks prior to TB-diagnosis, with detection rates increasing as sample collection times approached approach TB diagnosis. Most positive samples were obtained from HIV-infected children who were analyzed more frequently and might exhibit greater antigenemia due to reduced $M t b$ containment, but the utility of CFP-10 pep as a biomarker for initial $M t b$ infection or nascent TB deserves further study.

Current treatment guidelines indicate that drug susceptible TB cases should receive 6-9 months of anti-TB therapy, but treatment responses may vary with age, immune function, infection site, and drug resistance [27]. Overtreatment can increase adverse events, while inadequate treatment can promote TB recurrence and drug resistance. Rapid monitoring assays are needed to address these issues, but current microbiologic and immunologic assays lack required speed, sensitivity, and/or specificity, while Xpert MTB/RIF cannot distinguish live and dead mycobacteria, limiting its utility for treatment monitoring [28]. CFP-10pep detection rates and concentrations decreased after anti-TB treatment initiation in TB cases with clinical responses, suggesting CFP-10pep may serve as a biomarker of treatment response. Serum 
CFP-10pep evaluation would be particularly beneficial for groups, including infants, where sputum-derived results for diagnosis and treatment evaluation are rarely available.

This study has several limitations. First, it used cryopreserved serum from a study not designed to evaluate $\mathrm{TB}$ diagnostics. However, all suspected TB cases underwent extensive evaluation that allowed post-hoc assignment of current TB classifications, and longitudinal assessment pre- and post-TB evaluation. Serum samples were stored at $-80^{\circ} \mathrm{C}$ for 9 to 13 years prior to CFP-10pep assay analysis. We have previously reported that CFP-10pep signal does not markedly decrease after 30 days storage at $80{ }^{\circ} \mathrm{C}(90.7 \pm 0.6 \%$ recovery) [ 23]. We do not, however, have data for the effect of long-term storage, which might allow CFP-10 degradation or modification to attenuate CFP-10pep detection, and underestimate the diagnostic sensitivity of the CFP-10pep assay.

Second, our study exclusion criteria may have introduced bias affecting our sensitivity and specificity estimates. Most differences between the excluded and analyzed groups were minor (Table S1) but analyzed children were more likely to have been breast-fed, had less severe HIV and CD4 classifications, and had a higher overall death rate without prior TB diagnosis, although the fraction of HIV-infected children was larger in the analyzed versus excluded population. HIV-uninfected children may have preferentially excluded for reduced sample availability, since they had fewer study visits than HIV-infected children after the intervention period (24 weeks vs 12 weeks) and thus fewer visits at which serum could be drawn and archived for subsequent analysis. Excluded HIV-uninfected children may also have been healthier overall and thus have had fewer serum samples collected overall. Notably, though, TB diagnosis frequency was higher in the analyzed versus the excluded cohort for both the HIV-infected and HIV-uninfected children.

Third, the analyzed cohort contained relatively few TB cases, most of which were diagnosed as unconfirmed TB (92.3\%), reducing its ability to provide narrow confidence intervals for diagnostic sensitivity estimates, particularly for subgroup analyses. Several factors could explain the scarcity of confirmed TB cases. Infants and young children with $\mathrm{TB}$ frequently have paucibacillary $\mathrm{TB}$ and are difficult to diagnose by microbiologic methods. Our analysis cohort was also drawn from a study that employed active case finding, which can diagnose TB earlier than passive screening, often before such cases have positive microbiologic test results [29]. Our exclusion criteria did not markedly affect confirmed TB case frequency in our analyzed study population, however, since the frequency of such cases was similar in the overall group and our analyzed cohort (1.9\% vs. $1.6 \%)$.
Forth, this study cannot compare serum CFP-10pep results to molecular methods (e.g., Xpert) that were not available during the initial study. However, Xpert MTB/ RIF and Xpert MTB/RIF Ultra are not superior to $M t b$ culture for pediatric TB diagnosis [10, 30].Serum CFP10pep also had similar sensitivity for all TB manifestations, including HIV/TB co-infection and extrapulmonary $\mathrm{TB}$, in contrast to Xpert MTB/RIF which exhibits reduced sensitivity for these cases, although direct comparisons are still required to validate this difference.

Fifth, most children did not have serum available at their initial TB diagnosis or most of their P1041 study visits. We therefore estimated the diagnostic sensitivity of serum CFP-10pep by evaluating its detection rate in sera collected within \pm 24 weeks of $\mathrm{TB}$ diagnosis $( \pm 1$ HIV-uninfected cohort post-intervention study visit) to increase the number of TB cases with available sample. However, this approach is expected to underestimate diagnostic sensitivity, by evaluating some samples collected before TB development and others collected after anti-TB treatment responses. Similarly, the specificity estimate required all samples be CFP-10-negative and likely underestimate the specificity that would be obtained using a single sample. The lack of comprehensive serum samples at all study visits also required that evaluation of CFP-10pep sensitivity, and CFP-10pep decreases following anti-TB treatment, employ aggregate data from all cases instead of sequential data from the same cases. However, we are not aware of any other study with longitudinal samples and diagnostic information in a similar at-risk TB population that would allow such analyses.

Finally, the serum CFP-10pep assay described in this study utilizes MALDI-TOF mass spectrometry for its readout, which may constrain its utility in resource limited settings. Serum CFP-10pep analysis approximates or exceeds the sensitivity and specificity requirements of the WHO target product profile for non-sputum-based TB diagnostics, but does not address end-user, cost, speed, or infrastructure requirements of this profile in its current incarnation. Current research focused on the development of less expensive and complicated portable mass spectrometers could allow assays to be performed in settings lacking extensive infrastructure or highly trained personnel. Alternately, central laboratory networks similar to those developed to allow Xpert MTB/ RIF analyses in high endemic TB regions could increase access, particularly if simple on-site sample processing was employed to reduce shipping constraints.

This study evaluated diagnostic performance in a population of HIV-exposed infants born in a region with high TB incidence, who were therefore at high-risk for Mtb infection, rapid TB disease progression, and reinfection. It is not clear if this may have affected the diagnostic performance of our assay. Our data suggest 
that HIV-infection status did not markedly affect assay sensitivity and specificity, although the number of $\mathrm{TB}$ cases was too small to permit accurate evaluation of any such difference. It is also unclear if the cohort exclusion criteria, which disproportionally excluded HIVuninfected children while increasing the relative percentage of TB cases independent of HIV status, introduced a bias for sicker children that may have increased the apparent sensitivity of our assay.

However, despite the listed concerns about factors that could increase or decrease assay sensitivity, our assay data indicate that serum CFP-10pep detection has robust diagnostic sensitivity for TB in very young children, including paucibacillary and early TB cases missed by microbiologic assays, and has robust specificity to exclude at-risk children without TB, including children with latent TB infection. Given the ability of this assay to address these unmet diagnostic needs, future studies are warranted to refine the diagnostic performance of this assay in additional cohorts that address these concerns.

\section{Conclusions}

CFP-10 secretion is critical for $M t b$ pathogenesis [17], but its detection may be masked by its low abundance, protein interactions, or homology with related mycobacteria [18]. This study quantitates an immune-enriched CFP-10-spectic peptide by mass spectrometry after serum samples are digested to disrupt masking interactions [18, 22, 23]. Our results suggest that serum CFP10pep signal could improve TB diagnosis in children, as it exceeds the WHO-specified sensitivity requirements for new non-sputum diagnostics, and exhibits similar performance for all TB manifestations, including culture-negative TB, HIV/TB co-infection, and extrapulmonary $\mathrm{TB}$, which are normally challenging to diagnose. Our results also suggest that serum CFP-10pep analysis may have utility for diagnosis of nascent $\mathrm{TB}$ cases and TB treatment monitoring. Further analyses to validate these findings will require new study cohorts designed for biomarker analysis that employ consistent serial serum collection in well characterized patient populations. However, we believe that the apparent ability of CFP-10pep assay to directly predict, diagnose, and evaluate the therapeutic response of TB cases that are not detected and cannot be accurately monitored by available laboratory tests has great potential value for clinicians.

\section{Abbreviations}

CFP-10pep: CFP-10 peptide; INH: Isoniazid; IS: Internal standard; MALDI-

TOF: Matrix-assisted laser desorption ionization time-of-flight;

Mtb: Mycobacterium tuberculosis; NTM: Non-tuberculous mycobacteria;

TB: Tuberculosis; TST: Tuberculin skin tests; WHO: World Health Organization

\section{Supplementary Information}

The online version contains supplementary material available at https:/doi. org/10.1186/s12916-021-01983-w.
Additional file 1: Table S1. Comparison of the baseline demographics and clinical characteristics of P1041 participants excluded and included for evaluation by the serum CFP-10pep assay. Table S2. The distribution of participants who received INH versus placebo by HIV status and TB category. Table S3. Summary of sera available per child and their distribution by group. Table S4. Sera distribution by group and time to TB diagnosis or diagnosis window. Table S5. Distribution of Unconfirmed TB evidence among included/excluded infants. Table S6. Distribution of Unconfirmed TB evidence and CFP-10pep assay positivity rates. Table S7. CFP-10pep sensitivity among children who received INH versus placebo by HIV and Mtb infection status. Table S8. CFP-10pep specificity among children who received INH versus placebo by HIV and Mtb infection status. Table S9. Comparision of the diagnostic subgroups and classification criteria used in the P1041 protocol and the $2015 \mathrm{NIH}$ pediatric TB guidelines. Table S10. Cross-classification of analyzed children by P1041 and $2015 \mathrm{NIH}$ criteria. Fig. S1. Age distribution at time of TB diagnosis. Fig. S2. Venn diagrams for the availability and TB diagnostic contribution of clinical data. Fig. S3. Serum CFP-10pep signal distribution by HIV infection status and TB diagnosis category and distribution of positive TB diagnostic results and CPF-10pep signal relative to TB diagnosis. Fig. S4. Positive proportions of CFP-10pep, culture and smear results relative to TB diagnosis time. Fig. S5. Changes in serum CFP-10pep levels from pre-diagnosis to post-treatment initiation in representative TB cases.

\section{Acknowledgements}

The authors express their gratitude to staff of Center for Cellular and Molecular Diagnosis of Tulane University for their support and advice in the conduct of this study.

\section{Authors' contributions}

The study was designed by C.D.M, and T.Y.H. Data analysis and interpretation was performed by L.M., S.K., and B. N, D.B. Manuscript design and editing was conducted by L.M., S.L., C.L., J.F., C.J.L., S.K., Z.S., S.N., C. M, and T.Y.H. The authors read and approved the final manuscript.

\section{Funding}

The funders had no role in the design, collection of data, data analysis, and interpretation or decision to submit the manuscript to this journal. The corresponding authors had full access to all study data and along with the principal investigators had final responsibility for the decision to submit for publication. This study was supported by R01Al113725, R01Al122932, and R21Al126361 (T.H.) and K23Al120793 and R21Al143341 (S.L.) from the National Institute of Allergy and Infectious Diseases (NIAID) and R01HD090927 from the Eunice Kennedy Shriver National Institute of Child Health and Human Development (NICHD) and W8IXWH1910926 from the Department of Defense (T.H.). Overall support for the International Maternal Pediatric Adolescent AIDS Clinical Trials Network (IMPAACT) was provided by the NIAID with co-funding from the NICHD and the National Institute of Mental Health, all components of the National Institutes of Health, under Award Numbers UM1AI068632 (IMPAACT LOC), UM1AI068616 (IMPAACT SDMC), and UM1Al106716 (IMPAACT LC), and by NICHD contract number HHSN275201800001I. We thank the parents and legal guardians for allowing their children to participate in the parent clinical trial, the health care workers for providing care to the participants, and other members of the P1041 team for assisting in the conduct of the study.

\section{Availability of data and materials}

The datasets used and analyzed during the current study are available from the corresponding author on reasonable request.

\section{Declarations}

\section{Ethics approval and consent to participate}

This study was approved by the institutional review board of University of Miami (HSRO20040353). Written informed consent was obtained from the legal guardians of the children in parent study (IMPAACT P1041 study, ClinicalTrials.gov number, NCT0008119). 


\section{Consent for publication}

Not applicable.

\section{Competing interests}

Y. H, C.L. and C.J.L. report other support from NanoPin Technologies, Inc., outside the submitted work; In addition, Y. H. has a patent "Compositions and methods of determining a level of active Mycobacterium tuberculosis infectious in a subject" licensed to NanoPin Technologies, Inc. Dr. Liu has patents PCT/US18/15742 and PCT/US18/16115 with royalties paid to NanoPin Technologies Inc.. All other authors declare no competing interests.

\section{Author details}

${ }^{1}$ Center for Cellular and Molecular Diagnostics, Biochemistry and Molecular Biology, Tulane University School of Medicine, Room 474, 333 S. Liberty Street, New Orleans, LA 70112, USA. ²Department of Laboratory Medicine, Tongji Hospital, Tongji Medical College, Huazhong University of Science and Technology, Wuhan 430030, China. ${ }^{3}$ Departments of Medicine and Global Health, Division of Allergy and Infectious Diseases, University of Washington, Seattle, WA 98104, USA. ${ }^{4}$ Frontier Science Foundation, Brookline, MA 02115, USA. ${ }^{5}$ Department of Chemical Engineering, Biomedical Engineering Program, University of South Carolina, Columbia, SC 29208, USA. ${ }^{6}$ Department of Pediatrics, State University of New York at Stony Brook, Stony Brook, NY 11794, USA. 'Department of Pediatrics, Division of Infectious Diseases and Immunology, University of Miami Miller School of Medicine, Batchelor Children's Research Institute, Room 286, 1580 NW 10th Avenue, Miami, FL 33136, USA.

Received: 22 January 2021 Accepted: 7 April 2021 Published online: 18 May 2021

\section{References}

1. World Health Organization. Global tuberculosis report 2019. https://apps. who.int/iris/handle/10665/329368. Assessed 15 Jan 2020.

2. Dodd PJ, Yuen CM, Sismanidis C, et al. The global burden of tuberculosis mortality in children: a mathematical modeling study. Lancet Glob Health. 2017:5:e898-906

3. Perez-Velez CM, Marais BJ. Tuberculosis in children. N Engl J Med. 2012;367: 348-61.

4. Marais BJ, Gie RP, Hesseling AC, et al. A refined symptom-based approach to diagnose pulmonary tuberculosis in children. Pediatrics. 2006;118:e1350-9.

5. Edwards DJ, Kitetele F, Van Rie A. Agreement between clinical scoring systems used for the diagnosis of pediatric tuberculosis in the HIV era. Int J Tuberc Lung Dis. 2007;11:263-9.

6. Zar HJ, Connell TG, Nicol M. Diagnosis of pulmonary tuberculosis in children: new advances. Expert Rev Anti-Infect Ther. 2010;8:277-88.

7. Nicol MP, Zar HJ. New specimens and laboratory diagnostics for childhood pulmonary TB: progress and prospects. Paediatr Respir Rev. 2011;12:16-21.

8. Zar HJ, Hanslo D, Apolles $P$, et al. Induced sputum versus gastric lavage for microbiological confirmation of pulmonary tuberculosis in infants and young children: a prospective study. Lancet. 2005;365:130-4.

9. Pai M, Behr MA, Dowdy D, et al. Tuberculosis. Nat Rev Dis Primers. 2016;2: 16076.

10. World Health Organization. Automated real-time nucleic acid amplification technology for rapid and simultaneous detection of tuberculosis and rifampicin resistance: Xpert MTB/RIF assay for the diagnosis of pulmonary and extrapulmonary TB in adults and children: policy update. World Health Organization, 2013. https://apps.who.int/iris/handle/10665/112472. Assessed 15 Jan 2020

11. Detjen AK, DiNardo AR, Leyden J, et al. Xpert MTB/RIF assay for the diagnosis of pulmonary tuberculosis in children: a systematic review and meta-analysis. Lancet Respir Med. 2015;3:451-61.

12. Sabi I, Rachow A, Mapamba D, et al. Xpert MTB/RIF ultra assay for the diagnosis of pulmonary tuberculosis in children: a multicentre comparative accuracy study. J Inf Secur. 2018;77:321-7.

13. Nicol MP, Workman L, Prins M, et al. Accuracy of Xpert Mtb/Rif ultra for the diagnosis of pulmonary tuberculosis in children. Pediatr Infect Dis J. 2018;37:e261-3.

14. Atherton RR, Cresswell FV, Ellis J, et al. Xpert MTB/RIF ultra for tuberculosis testing in children: a mini-review and commentary. Front Pediatr. 2019;7:34.
15. Kampmann B, Whittaker E, Williams A, et al. Interferon- release assays do not identify more children with active tuberculosis than the tuberculin skin test. Eur Respir J. 2009;33:1374-82.

16. Furin J, Cox H, Pai M. Tuberculosis. Lancet. 2019;393:1642-56.

17. Kristi M, Guinn MJH, Mathur SK, Zakel KL, Grotzke JE, Lewinsohn DM, et al. Individual RD1-region genes are required for export of ESAT-6/CFP-10 and for virulence of Mycobacterium tuberculosis. Mol Microbiol. 2004;51:359-70.

18. Fan J, Zhang H, Nguyen DT, et al. Rapid diagnosis of new and relapse tuberculosis by quantification of a circulating antigen in HIV-infected adults in the Greater Houston metropolitan area. BMC Med. 2017;15:188.

19. Calligaro GL, Zijenah LS, Peter JG, et al. Effect of new tuberculosis diagnostic technologies on community-based intensified case finding: a multicentre randomized controlled trial. Lancet Infect Dis. 2017;17:441-50,

20. Brennan PJ. Structure, function, and biogenesis of the cell wall of Mycobacterium tuberculosis. Tuberculosis. 2003;83:91-7.

21. Flores LL, Steingart KR, Dendukuri N, et al. Systematic review and metaanalysis of antigen detection tests for the diagnosis of tuberculosis. Clin Vaccine Immunol. 2011;18:1616-27.

22. Liu C, Zhao Z, Fan J, et al. Quantification of circulating Mycobacterium tuberculosis antigen peptides allows rapid diagnosis of active disease and treatment monitoring. Proc Natl Acad Sci. 2017;114:3969-74.

23. Liu C, Lyon CJ, Bu Y, et al. Clinical evaluation of a blood assay to diagnose paucibacillary tuberculosis via bacterial antigens. Clin Chem. 2018:64:791-800.

24. Madhi SA, Nachman S, Violari A, et al. Primary isoniazid prophylaxis against tuberculosis in HIV-exposed children. N Engl J Med. 2011:365:21-31.

25. Graham SM, Cuevas LE, Jean-Philippe $P$, et al. Clinical case definitions for classification of intrathoracic tuberculosis in children: an update. Clin Infect Dis. 2015;61:S179-87.

26. World Health Organization. High-priority target product profiles for new tuberculosis diagnostics: report of a consensus meeting, 28-29 April 2014. Geneva: World Health Organization; 2014. https://appswhoint/iris/handle/1 0665/135617 Assessed 15 Jan 2020

27. Mahomed S, Padayatchi N, Singh J, et al. Precision medicine in resistant tuberculosis: treat the correct patient, at the correct time, with the correct drug. J Inf Secur. 2019;78:261-8.

28. Friedrich SO, Rachow A, Saathoff E, et al. Assessment of the sensitivity and specificity of Xpert MTB/RIF assay as an early sputum biomarker of response to tuberculosis treatment. Lancet Respir Med. 2013;1:462-70.

29. Saunders MJ, Tovar MA, Collier D, et al. Active and passive case-finding in tuberculosis-affected households in Peru: a 10-year prospective cohort study. Lancet Infect Dis. 2019;19:519-28.

30. World Health Organization. Guidance for national tuberculosis programmes on the management of tuberculosis in children. 2nd ed. https://apps.who. int/iris/handle/10665/112360. Assessed 15 Jan 2020

\section{Publisher's Note}

Springer Nature remains neutral with regard to jurisdictional claims in published maps and institutional affiliations.

Ready to submit your research? Choose BMC and benefit from:

- fast, convenient online submission

- thorough peer review by experienced researchers in your field

- rapid publication on acceptance

- support for research data, including large and complex data types

- gold Open Access which fosters wider collaboration and increased citations

- maximum visibility for your research: over $100 \mathrm{M}$ website views per year

At BMC, research is always in progress.

Learn more biomedcentral.com/submissions 